\title{
Real-time observation of collective excitations in photoemission
}

\author{
C. Lemell,,${ }^{1, *}$ S. Neppl, ${ }^{2}$ G. Wachter, ${ }^{1}$ K. Tókési,${ }^{3}$ R. Ernstorfer, ${ }^{4}$ P. Feulner, ${ }^{5}$ R. Kienberger,${ }^{6,7}$ and J. Burgdörfer ${ }^{1,3}$ \\ ${ }^{1}$ Institute for Theoretical Physics, Vienna University of Technology, 1040 Vienna, Austria, EU \\ ${ }^{2}$ Lawrence Berkeley National Laboratory, Chemical Sciences Division, Berkeley, California 94720, USA \\ ${ }^{3}$ Institute for Nuclear Research, Hungarian Academy of Sciences (ATOMKI), 4001 Debrecen, Hungary, EU \\ ${ }^{4}$ Fritz-Haber-Institut der Max-Planck-Gesellschaft, 14195 Berlin, Germany, EU \\ ${ }^{5}$ Physikdepartment E20, Technische Universität München, 85747 Garching, Germany, EU \\ ${ }^{6}$ Physikdepartment E11, Technische Universität München, 85747 Garching, Germany, EU \\ ${ }^{7}$ Max-Planck-Insitut für Quantenoptik, Hans Kopfermann Straße 1, 85748 Garching, Germany, EU \\ (Received 12 January 2015; revised manuscript received 13 May 2015; published 3 June 2015)
}

\begin{abstract}
Ejection of an electron by absorption of an extreme ultraviolet (xuv) photon probes the many-electron response of a solid well beyond the single-particle picture. Photoemission spectra feature complex correlation satellite structures signifying the simultaneous excitation of single or multiple plasmons. The time delay of the plasmon satellites relative to the main line can be resolved in attosecond streaking experiments. Time-resolved photoemission thus provides the key to discriminate between intrinsic and extrinsic plasmon excitation. We demonstrate the determination of the branching ratio between intrinsic and extrinsic plasmon generation for simple metals.
\end{abstract}

DOI: 10.1103/PhysRevB.91.241101

PACS number(s): 78.47.J-, 68.35.Ja, 71.45.Gm, 79.60.-i

Time-resolved photoemission from solid surfaces by attosecond streaking [1] pioneered by Cavalieri et al. [2] allows one to not only probe electron dynamics near surfaces on its natural time scale (typical traveling times of photoelectrons in the material $\sim 100$ as) but also features high surface sensitivity on the few-angstrom scale (screening length of the streaking field $\sim 1 \AA$ ) [3-11]. This unprecedented spatiotemporal resolution promises novel insights into manybody and correlation effects present in photoemission. Deconvolution of the processes underlying photoemission from interacting many-electron systems has remained a challenge in view of the large number of degrees of freedom involved. Thus, attosecond chronoscopy promises to provide novel information complementary to conventional photoelectron spectroscopy.

The starting point of a quantum description of photoemission in lowest-order perturbation theory in the ionizing xuv field is the so-called "one-step" or multiple-scattering model $[12,13]$ in which the response of the solid to the photoabsorption is represented by a coherent superposition of a set of stationary states of the $(N-1)$-electron system and a wave packet of the emitted electron formed by oneelectron scattering states [13]. The set of accessible final states of the $(N-1)$-electron system includes a large number of complex dynamical processes. In standard photoemission spectroscopy, the degrees of freedom associated with the residual ionic complex remain, in general, unobserved. Information on a multitude of many-body effects such as, e.g., core-hole screening $[14,15]$ and relaxation, particle-hole and plasmon excitation is thus encoded in the reduced oneelectron photoemission spectrum. To disentangle underlying processes a simplified "three-step" model of photoemission [13] is frequently employed in which the response of the many-body state is reduced to few active degrees of freedom with which the outgoing photoelectron interacts. In this

*1emell@ concord.itp.tuwien.ac.at model, the photoemission process by an xuv photon is broken down into a sequence of elementary processes: the primary photoabsorption transferring a target electron to a high-lying quasicontinuum state in the conduction band, the transport of the resulting Bloch wave packet towards the surface subject to elastic and inelastic scattering, and, finally, diffraction at the surface potential and eventual escape from the solid with asymptotic energy $E=k^{2} / 2$ (atomic units are used unless otherwise stated). This multistep model suggests time ordering of these elementary processes.

In this Rapid Communication we aim at observing in real time the unfolding of this multistep scenario using the attosecond streaking technique. Combining spectral and temporal information for each line of the photoelectron spectrum separately allows one to disentangle energy-degenerate channels. We demonstrate the potential of time-resolved photoemission from surfaces for a prototypical nearly-free-electron metal, magnesium. Here, the many-electron response is well approximated by a small number of primary excitation and postexcitation scattering channels. Accordingly, the energy of the absorbed photon can be either transferred entirely to the photoelectron giving rise to the main line in the spectrum at $E=\hbar \omega_{\text {xuv }}-E_{\text {bind }}$ or can simultaneously excite "intrinsic" plasmons due to the interaction of the electron gas with the core hole resulting in satellite lines $E=\hbar \omega_{\text {xuv }}-E_{\text {bind }}-n \hbar \omega_{\mathrm{pl}}$ with $n$ the number of collective excitations in the conduction band. This process originally proposed by Lundquist [16] and Penn [17,18] can be viewed as a condensed-matter analog to the shake-up (or correlation) satellites in atomic photoionization [19-21].

An alternative scenario for plasmon excitation is inelastic scattering of the primary energetic photoelectron on its path towards the surface giving rise to particle-hole or collective excitations. The latter case is referred to as "extrinsic" plasmon excitation and is often considered a background contribution. As the final state of the photoelectron is energetically degenerate with that accompanied by intrinsic plasmon excitation, the distinction between extrinsic and 

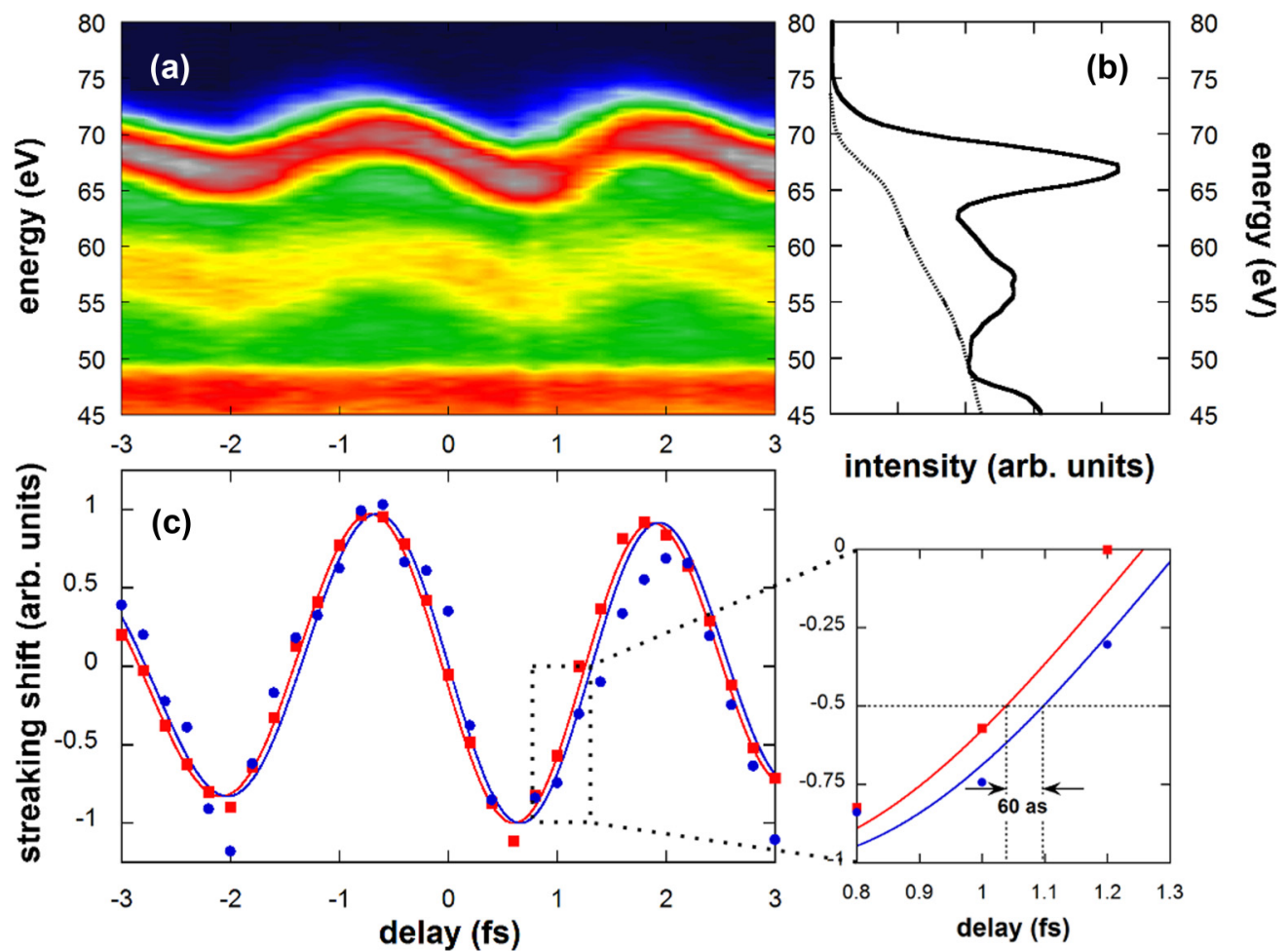

FIG. 1. (Color online) (a) Experimental streaking traces of the $\mathrm{Mg} 2 p$ and plasmon-satellite lines; (c) relative shift of the streaking traces (symbols: experimental data; lines: best fit) of about $60 \pm 10$ as can be extracted (zoom-in). (b) Uncorrected NIR-field free photoelectron spectrum and the standard background subtraction [25] are shown. Electron energies are given throughout the text relative to the Fermi energy $E_{F}$.

intrinsic plasmon excitation in photoelectron spectra is far from clear cut. The disparity of the characteristic length scale of the intrinsic process given by the screening length $a_{\mathrm{sc}}$ of the core hole $(\sim 1.5 \AA$ in $\mathrm{Mg})$ and the corresponding length scale for extrinsic plasmon loss, the inelastic mean free path ( $\lambda_{\text {inel }} \sim 5 \AA$ in the present experiment) suggests the possibility to distinguish these processes. Determination of the fraction $\alpha$ of intrinsic plasmon excitation accompanying photoemission by conventional photospectroscopy has remained a challenge. Theoretical and experimental estimates vary widely between $0 \%$ and $40 \%$ (see [22], and references therein) with theoretical models $[23,24]$ expecting a strong dependence on the energy of the released photoelectron. For the absorption of xuv photons in magnesium in the spectral range considered here ( $\hbar \omega \sim 120 \mathrm{eV}$ ) a vanishing probability for intrinsic plasmon excitation has been predicted. The uncertainties in $\alpha$ result from the difficulty in distinguishing extrinsic from intrinsic plasmons and from ambiguities related to the inevitable background subtraction [25]. Within the multistep scenario of photoemission, extrinsic plasmon excitation occurring in the transport step is time delayed relative to intrinsic plasmon excitation occurring in the primary photoabsorption step. Therefore, attosecond chronoscopy may provide the key to disentangle extrinsic and intrinsic plasmon satellites and, on a more fundamental level, to scrutinize the validity of such a multistep description of photoemission for condensed matter.

In our experiment (for details see [26,27]), a linearly polarized xuv laser pulse with a central energy of $118 \mathrm{eV}$ induces photoemission from a $\mathrm{Mg}(0001)$ surface. Collinear to it, a 4.5-fs-long near-infrared (NIR) laser pulse streaks the photoelectrons. Streaking traces of the emission lines from the $\mathrm{Mg} 2 p$ core level $\left(E_{\text {bind }} \approx 50 \mathrm{eV}\right)$ and from the conduction band are accompanied by satellite lines at energies reduced by $\hbar \omega_{\mathrm{pl}} \approx 10.5 \mathrm{eV}$ relative to the main line. Here we analyze the streaking traces of the $\mathrm{Mg} 2 p$ photoelectron line and its single plasmon-loss satellite (Fig. 1). The modulation in emission energy is determined by the momentum shift $\Delta p(\tau)=A(\tau)$ the NIR streaking field imparts on the escaping electron. $A(t)$ denotes the vector potential of the laser pulse and $\tau$ the moment of arrival of the photoelectron in the streaking field. Analyzing the first moments of these emission lines as a function of the xuv-NIR delay reveals a relative streaking delay $\Delta \tau_{s}=\tau[2 p+\mathrm{pl}]-\tau[2 p]=60 \pm 10$ as of the plasmon satellite line (for details see, e.g., [27]).

One key to the extraction of timing information from attosecond streaking of condensed-matter systems is the penetration depth and dielectric screening of the NIR field at the surface with angstrom accuracy. We employ time-dependent density functional theory (TDDFT) to simulate the formation of the polarization charge layer at the surface. Our results show that a screening charge is formed with its centroid at $z_{\mathrm{c}} \approx 3.5$ a.u. above the topmost atomic layer which determines the effective position of the (electronic) surface. The screening distance for the NIR field is found to be $d \approx 2.5$ a.u. Thus, photoabsorption and formation of the Bloch wave packet take place fully screened from the streaking pulse. Only upon crossing the metal-vacuum interface, the photoelectron is exposed to the NIR field [27]. 
We simulate the photoemission process by the three-step model employing a variant of the semiclassical transport theory described in more detail elsewhere [3]. Accordingly, the location of the primary photoabsorption event is randomly distributed over the topmost 15 layers with a probability density deduced from the layer-averaged ground-state DFT electron-density distribution. The stochastic ionization time distribution follows the Gaussian temporal intensity distribution of the xuv pulse with an FWHM of the intensity of $\tau_{\mathrm{xuv}}=450$ as. The mean energy of the Bloch wave packet following direct photoexcitation of the $\mathrm{Mg} 2 p$ core electron is $E[2 p]=\hbar \omega_{\text {xuv }}-E_{\text {bind }} \approx 68 \mathrm{eV}$ with width $\Gamma_{2 p} \simeq$ $\Gamma_{\mathrm{xuv}}=\pi / \tau_{\mathrm{xuv}} \approx 4.5 \mathrm{eV}$, while the ionization accompanied by intrinsic plasmon (ipl) excitation results in an energy-shifted wave packet ("plasmon satellite") at $E[2 p+\mathrm{ipl}]=\hbar \omega_{\text {xuv }}-$ $E_{\text {bind }}-\hbar \omega_{\mathrm{pl}} \approx 58 \mathrm{eV}$ with $\Gamma \simeq \Gamma_{\text {xuv }}+\Gamma_{\mathrm{pl}} \approx 6.5 \mathrm{eV}$. Because of the lack of accurate microscopic predictions for the fraction $\alpha$,

$$
\alpha=\frac{I_{0}[2 p+\mathrm{ipl}]}{I_{0}[2 p]+I_{0}[2 p+\mathrm{ipl}]},
$$

where $I_{0}[2 p+\mathrm{ipl}]$ and $I_{0}[2 p]$ are the initial intensities for photoexcitation with and without an accompanying intrinsic plasmon excitation, we treat $\alpha$ as an adjustable input parameter of our simulation to be determined by comparison with the observed streaking delay. Multiple plasmon excitation is expected to be weak and is neglected in the following.

The primary wave packets of the main line and the intrinsic plasmon satellite are propagated through the crystal subject to inelastic and elastic [28] scattering until they either escape through the surface or are slowed down to energies $(E \leqslant 40 \mathrm{eV})$ below the detection window. Inelastic scattering is determined from the loss function $-\operatorname{Im}\left[\varepsilon^{-1}(q, \omega)\right]$ for Mg proportional to $d^{2} \lambda(E)_{\text {inel }}^{-1} / d \omega d q$, the doubly differential inverse inelastic mean free path for a quasi-free electron with kinetic energy $E=k^{2} / 2$ (the effective mass of the conduction band electron in $\mathrm{Mg}$ is close to 1 a.u. [27]). $d^{2} \lambda(E)_{\text {inel }}^{-1} / d \omega d q$ determines the probability density for the collisional energy $(\omega)$ and momentum $(q)$ transfers as well as the inelastic mean free path $\lambda_{\text {inel }}(E)$ between two subsequent inelastic collision events treated as a stochastic Poisson process. We model $-\operatorname{Im}\left[\varepsilon^{-1}(q, \omega)\right]$ for the (almost) free-electron-gas metal $\mathrm{Mg}$ using the analytical loss function of Mermin [29,30] [Fig. 2(a)] with parameters derived from comparison with photoabsorption data ["optical data" [31], $-\operatorname{Im}\left[\varepsilon^{-1}(q=0, \omega)\right]$; Fig. 2(b)].

The dominant feature in $-\operatorname{Im}\left[\varepsilon^{-1}(q=0, \omega)\right]$ is the plasmon loss peak. The resulting theoretical $\lambda_{\text {inel }}(E)$ agrees for the energy range presently considered with the NIST reference data [32-34] to within 5\%. The small $\lambda_{\text {inel }}(E)$ (less than two lattice spacings) derived from the bulk dielectric function point to the strong surface sensitivity of the photoemission process at xuv energies. In turn, the largest uncertainties in $\lambda_{\text {inel }}(E)$ result from surface effects because of the depletion of the electronic density in the vicinity of the surface and the onset of surface plasmon excitation [35-37] spectrally unresolved in the present experiment. We therefore expect $\lambda_{\text {inel }}(E)$ to be slightly enhanced near the surface compared to its bulk value.

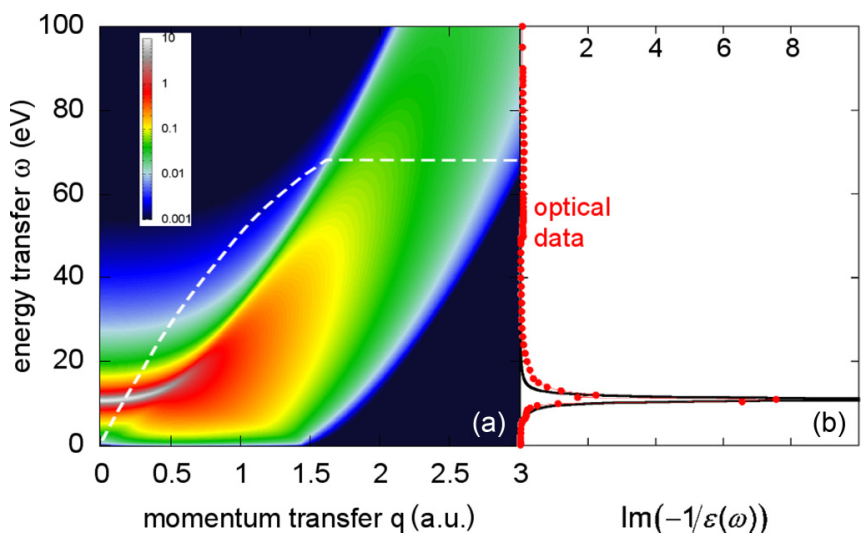

FIG. 2. (Color online) (a) Loss function $-\operatorname{Im}\left[\varepsilon^{-1}(q, \omega)\right]$ for a free-electron gas with density and width of the plasmon peak (logarithmic color scale) derived from optical data of $\mathrm{Mg}$. The dashed white line delimits the region accessible to inelastic scattering of an electron from the $\mathrm{Mg} 2 p$ peak satisfying energy and momentum conservation. (b) Photoabsorption data $\left(-\operatorname{Im}\left[\varepsilon^{-1}(q=0, \omega)\right]\right)$ for $\mathrm{Mg}$ (red circles [31]) and calculated optical data (black solid line, linear scale).

On its path towards the surface the wave packet may suffer a characteristic energy loss $\Delta E=\hbar \omega_{\mathrm{pl}}$ by exciting an extrinsic plasmon (epl) in an inelastic collision. As a result, the portion of the wave packet representing the main line undergoing one inelastic scattering process becomes energetically degenerate with the wave packet associated with the intrinsic plasmon-satellite line, $E[2 p+$ epl $] \simeq E[2 p+$ ipl $]$. While spectrally difficult to resolve, the two channels feature different mean transport lengths and, hence, different time delays relative to the xuv pulse. Neglecting, for the moment, the weak energy dependence of $\lambda_{\text {inel }}$ and other loss channels, a simple estimate for the streaking time delay can be given. In a Poissonian stochastic scattering sequence, the average distance traveled by the photoelectron without undergoing any inelastic scattering process contributing to the main line is $\lambda_{\text {inel }}$. Correspondingly, the mean distance traveled by electrons undergoing no more than one inelastic scattering appearing in the extrinsic one-plasmon satellite is $2 \lambda_{\text {inel }}$. Consequently, for exclusively extrinsic $(\alpha=0)$ plasmon excitation we expect an approximate streaking delay between the extrinsic plasmon line and the main line of $\Delta \tau=\lambda_{\text {inel }} / v \approx \lambda_{\text {inel }} / \sqrt{2 E} \approx 65$ as. In the opposite limit of intrinsic plasmon excitation (close to $\alpha=1$ ), this transport-related delay should vanish and the residual delay is given by the difference in intrinsic EisenbudWigner-Smith (EWS) delay $\Delta \tau_{s}=\Delta \tau_{\text {EWS }}$ between the main line and the intrinsic plasmon satellite which is expected to be small compared to the transport delay in line with the delay $\Delta \tau_{\text {EWS }}$ for atomic correlation satellites for which ab initio calculations yield only a few attoseconds [38].

The simulated mean escape-time distribution [Fig. 3(b)] confirms this simple analytic estimate: electrons which have excited an extrinsic plasmon along their trajectory acquire, on average, a delay relative to those that escape without undergoing an inelastic scattering event of the order of $\Delta \tau$. The streaking delay $\Delta \tau_{s}=\tau[2 p+\mathrm{pl}]-\tau[2 p]$ can now be expressed in terms of the mean delay for the $E[2 p+\mathrm{pl}]$ 


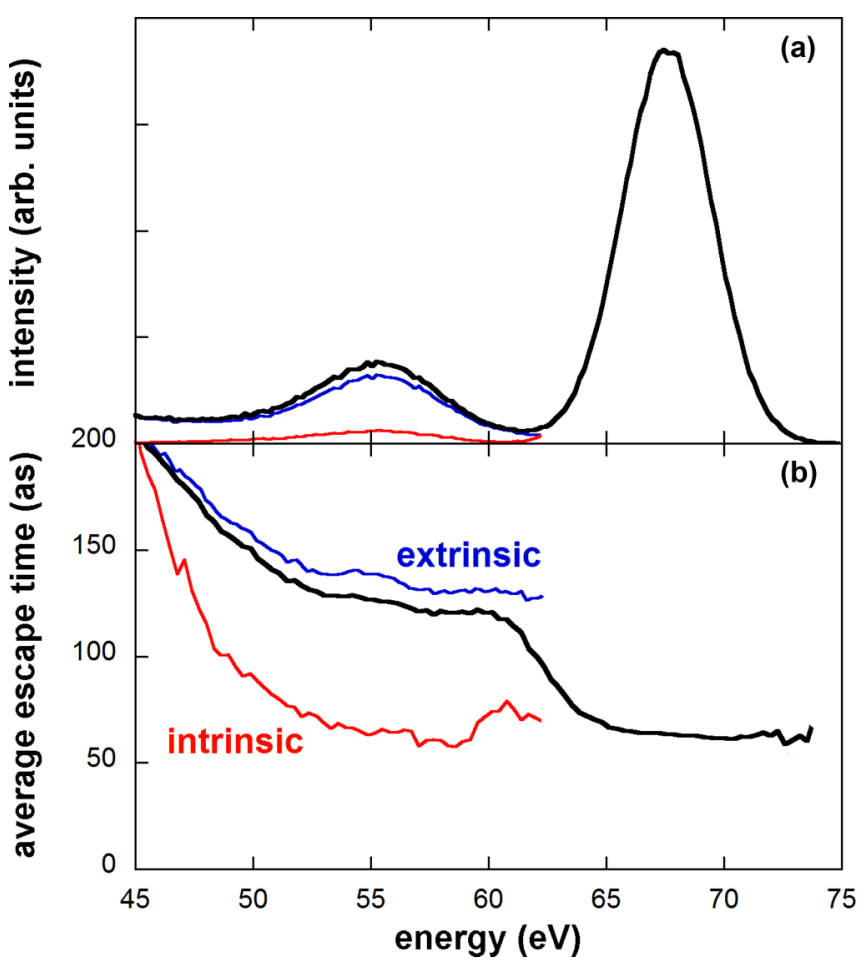

FIG. 3. (Color online) (a) Energy spectrum (thick black solid line) for $\alpha=0.05$ and (b) average escape time distribution for $\mathrm{Mg}$ $2 p$ photoelectrons excited by an $\hbar \omega_{\mathrm{xuv}}=118 \mathrm{eV}$ pulse. Intrinsic contributions (red) to the satellite peak, $I[2 p+\mathrm{ipl}]$; photoelectrons exciting an extrinsic plasmon (blue line) along their trajectory, $I[2 p+\mathrm{epl}]$.

electrons given by the weighted average

$$
\tau[2 p+\mathrm{pl}]=\frac{\tau_{\mathrm{ipl}} I[2 p+\mathrm{ipl}]+\tau_{\mathrm{epl}} I[2 p+\mathrm{epl}]}{I[2 p+\mathrm{pl}]},
$$

where $I[2 p+\mathrm{ipl}]$ and $I[2 p+\mathrm{epl}]$ depend on $\alpha$ and on the inelastic scattering process, i.e., on $\lambda_{\text {inel }}$. As the partial intensities also determine the experimental intensity ratio of the direct and plasmon-loss peaks,

$$
R=\frac{I[2 p]}{I[2 p+\mathrm{pl}]}=\frac{I[2 p]}{I[2 p+\mathrm{epl}]+I[2 p+\mathrm{ipl}]},
$$

the experimental values of $R$ and $\Delta \tau_{s}$ can be used to determine both $\alpha$ and the effective $\lambda_{\text {inel }}$ simultaneously. The extraction of the accurate value of $R$ depends sensitively on the details of the background subtraction. Applying the Shirley procedure [25] we find $R \approx 3$. Alternatively, assuming the main line to be background free and only the plasmon satellite is distorted by secondary electron-emission processes, $R$ increases to about 3.5. This window of admissible values of $R$ restricts the range of admissible values of $\alpha$ to $0.05 \leqslant \alpha \leqslant 0.15$ consistent with the streaking delay $\Delta \tau_{s}=60 \pm 10$ as (Fig. 4). Theoretical values of $\Delta \tau_{s}$ as a function of $\alpha$ agree with the experimental value $\Delta \tau_{s}=60$ as for $\alpha=0.05$ when using the bulk value by $\Delta \lambda$ in the transport simulation (lower red line in Fig. 4). A possible slight enhancement of $\lambda_{\text {inel }}$ near the surface relative to its bulk value, $\Delta \lambda$, retaining agreement between simulation and experiment is delimited by $\Delta \lambda \lesssim 1 \AA$ or less than $20 \%$ (upper red line in Fig. 4). The comparison between experiment

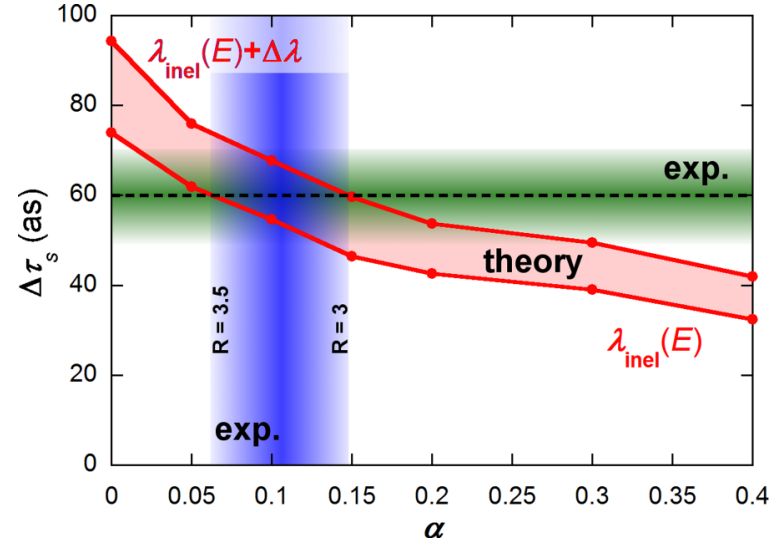

FIG. 4. (Color online) Comparison between experiment and simulation for photoemission from $\mathrm{Mg} 2 p$ accompanied by extrinsic or intrinsic plasmon excitation following the absorption of xuv photons with $\hbar \omega_{\text {xuv }}=118 \mathrm{eV}$ in the $\left(\Delta \tau_{s}, \alpha\right)$ plane. Horizontal (green) shaded area: experimental streaking time delay $\Delta \tau_{s}$ between the plasmon satellite $E[2 p+\mathrm{pl}]$ relative to the main line $E[2 p]$; vertical (blue) shaded area: allowed values of the intrinsic excitation probability $\alpha$ consistent with the experimental relative spectral intensity ratios $R$ [see Eq. (3)]; red shaded area: theoretically predicted dependence of $\Delta \tau_{s}(\alpha)$ for inelastic mean free paths varied in the interval $\left[\lambda_{\text {inel }}, \lambda_{\text {inel }}+\Delta \lambda\right]$ with $\Delta \lambda=1 \AA$ and theoretical $\lambda_{\text {inel }}$. Overlap of these three areas provides accurate estimates for $\left(\Delta \tau_{s}, \alpha\right)$.

and theory in the $\left(\Delta \tau_{s}, \alpha\right)$ plane (Fig. 4) thus provides the opportunity to simultaneously determine the intrinsic plasmon excitation rate $(\alpha=0.1 \pm 0.05)$ and the inelastic mean-freepath near surfaces $\left(\lambda_{\text {inel }}=5.5 \pm 0.5 \AA\right)$ with unprecedented accuracy. Combining temporal and spectral information, a small but finite probability for intrinsic plasmon excitation in a prototypical simple metal, magnesium, at photon energies of $\hbar \omega \approx 100 \mathrm{eV}$ well below the sudden shake-up regime can be unambiguously determined. The window of admissible fractions $\alpha$ for intrinsic plasmon-satellite excitation found is consistent to that of typical atomic shake-up excitation probabilities at comparable energies (e.g., $\mathrm{He} \sim 7 \%[39,40]$ ) providing a measure for electronic ground-state correlation in atoms.

In this work we have demonstrated the ability of attosecond chronoscopy to temporally resolve spectrally overlapping processes accompanying photoemission from solids. The measurement of streaking time shifts in combination with photoelectron spectra provides the key to disentangle emission channels that are energetically degenerate. For the prototypical case of the nearly-free-electron metal $\mathrm{Mg}$ we have demonstrated the deconvolution of the plasmon-loss peak in the electron-energy spectrum into intrinsic and extrinsic contributions. Contributions due to intrinsic and extrinsic plasmon excitation can be disentangled with remarkable accuracy. Concomitantly, the characteristic transport length can be probed with (sub)angstrom precision. This method holds the promise to probe the many-electron response in the photoemission from more complex and strongly correlated materials [41] in unprecedented detail.

This work has been supported within the FWF special research programs SFB-041 (ViCoM) and SFB-049 (NextLite), 
doctoral programme DK-W1243 (Solid4Fun), FWF project P21141-N16, and by the European COST Action CM1204 (XLIC). S.N., P.F., and R.K. were supported by the DFG through the Munich-Centre for Advanced Photonics projects C.1.5, C.1.8, and B.1.4. G.W. is supported by the International
Max Planck Research School on Advanced Photon Science (IMPRS-APS) of the Max Planck Gesellschaft. One of us (K.T.) was supported by the Hungarian Scientific research Fund OTKA project NN 103279. Supporting calculations have been performed on the Vienna Scientific Cluster.
[1] R. Kienberger, E. Goulielmakis, M. Uiberacker, A. Baltuska, V. Yakovlev, F. Bammer, A. Scrinzi, T. Westerwalbesloh, U. Kleineberg, U. Heinzmann, M. Drescher, and F. Krausz, Nature (London) 427, 817 (2004).

[2] A. L. Cavalieri, N. Müller, Th. Uphues, V. S. Yakovlev, A. Baltuska, B. Horvath, B. Schmidt, L. Blümel, R. Holzwarth, S. Hendel, M. Drescher, U. Kleineberg, P. M. Echenique, R. Kienberger, F. Krausz, and U. Heinzmann, Nature (London) 449, 1029 (2007).

[3] C. Lemell, B. Solleder, K. Tőkési, and J. Burgdörfer, Phys. Rev. A 79, 062901 (2009).

[4] A. K. Kazansky and P. M. Echenique, Phys. Rev. Lett. 102, 177401 (2009).

[5] C.-H. Zhang and U. Thumm, Phys. Rev. Lett. 102, 123601 (2009).

[6] C.-H. Zhang and U. Thumm, Phys. Rev. A 82, 043405 (2010).

[7] C.-H. Zhang and U. Thumm, Phys. Rev. A 84, 033401 (2011); 84, 063403 (2011); 84, 065403 (2011).

[8] E. E. Krasovskii, Phys. Rev. B 84, 195106 (2011).

[9] E. E. Krasovskii, V. M. Silkin, V. U. Nazarov, P. M. Echenique, and E. V. Chulkov, Phys. Rev. B 82, 125102 (2010).

[10] A. G. Borisov, D. Sanchez-Portal, A. K. Kazansky, and P. M. Echenique, Phys. Rev. B 87, 121110 (2013).

[11] J. D. Lee, Phys. Rev. Lett. 111, 027401 (2013).

[12] G. Mahan, Phys. Rev. B 2, 4334 (1970).

[13] P. Feibelman and D. Eastman, Phys. Rev. B 10, 4932 (1974).

[14] G. S. Canright, Phys. Rev. B 38, 1647 (1988).

[15] N. E. Koval, D. Sanchez-Portal, A. G. Borisov, and R. Diez Muino, Nanoscale Res. Lett. 7, 447 (2012).

[16] B. I. Lundqvist, Phys. Kondens. Mater. 9, 236 (1969).

[17] D. R. Penn, Phys. Rev. Lett. 38, 1429 (1977).

[18] D. R. Penn, Phys. Rev. Lett. 40, 568 (1978).

[19] F. W. Byron, Jr. and C. J. Joachain, Phys. Rev. 164, 1 (1967).

[20] T. Åberg, Phys. Rev. A 2, 1726 (1970).

[21] A. Dalgarno and H. R. Sadeghpour, Phys. Rev. A 46, R3591(R) (1992).

[22] M. Kurth, P. C. J. Graat, and E. J. Mittemeijer, Appl. Surf. Sci. 220, 60 (2003).
[23] J. W. Gadzuk, J. Electron Spectrosc. Relat. Phenom. 11, 355 (1977).

[24] D. Chastenet and P. Longe, Phys. Rev. Lett. 44, 91 (1980).

[25] D. A. Shirley, Phys. Rev. B 5, 4709 (1972).

[26] S. Neppl, R. Ernstorfer, E. M. Bothschafter, A. L. Cavalieri, D. Menzel, J. V. Barth, F. Krausz, R. Kienberger, and P. Feulner, Phys. Rev. Lett. 109, 087401 (2012).

[27] S. Neppl, R. Ernstorfer, A. L. Cavalieri, C. Lemell, G. Wachter, E. Magerl, E. M. Bothschafter, M. Jobst, M. Hofstetter, U. Kleineberg, J. V. Barth, D. Menzel, J. Burgdörfer, P. Feulner, F. Krausz, and R. Kienberger, Nature (London) 517, 342 (2015).

[28] F. Salvat, A. Jablonski, and C. Powell, Comput. Phys. Commun. 165, 157 (2005).

[29] N. D. Mermin, Phys. Rev. B 1, 2362 (1970).

[30] J. Lindhard, K. Dan. Vidensk. Selsk. Mat.-Fys. Medd. 28(8) (1954).

[31] Handbook of Optical Constants of Solids, edited by E. D. Palik (Academic, New York, 1991).

[32] C. J. Powell and A. Jablonski, NIST Electron Inelastic-MeanFree-Path Database, version 1.2, SRD71 (National Institute of Standards and Technology, Gaithersburg, MD, 2010).

[33] S. Tanuma, C. J. Powell, and D. R. Penn, Surf. Interface Anal. 43, 689 (2011).

[34] H. Shinotsuka, S. Tanuma, C. J. Powell, and D. R. Penn, Nucl. Instrum. Methods Phys. Res. B 270, 75 (2012).

[35] C. O. Reinhold and J. Burgdörfer, Phys. Rev. A 55, 450 (1997).

[36] Y. C. Li, Y. H. Tu, C. M. Kwei, and C. J. Tung, Surf. Sci. 589, 67 (2005)

[37] M. Novak, Surf. Sci. 602, 1458 (2008).

[38] R. Pazourek, J. Feist, S. Nagele, and J. Burgdörfer, Phys. Rev. Lett. 108, 163001 (2012).

[39] R. Wehlitz, I. A. Sellin, O. Hemmers, S. B. Whitfield, P. Glans, H. Wang, D. W. Lindle, B. Langer, N. Berrah, J. Viefhaus, and U. Becker, J. Phys. B: At., Mol. Opt. Phys. 30, L51 (1997).

[40] J.-Z. Tang and J. Burgdörfer, J. Phys. B: At., Mol. Opt. Phys. 30, L523 (1997).

[41] J. D. Lee, Phys. Rev. B 86, 035101 (2012). 